\title{
An Approach to Design Smart Grids and their IT System by Cosimulation
}

\author{
David Oudart $^{1,2}$, Jérôme Cantenot ${ }^{1}$, Frédéric Boulanger ${ }^{3}$ and Sophie Chabridon ${ }^{2}$ \\ ${ }^{1}$ EDF R\&D, Palaiseau, France \\ ${ }^{2}$ SAMOVAR, CNRS, Université Paris-Saclay, Télécom SudParis, Évry, France \\ ${ }^{3}$ LRI, CNRS, CentraleSupélec, Université Paris-Saclay, France
}

Keywords: Cosimulation, FMI, IT, MDE, smart grid, cyber-physical system

\begin{abstract}
Smart grids are the oncoming generation of power grids, which rely on information and communication technologies to tackle decentralized and intermittent energy sources such as wind farms and photovoltaic plants. They integrate electronics, software information processing and telecommunications technical domains. Therefore the design of smart grids is complex because of the various technical domains and modeling tools at stake. In this article, we present an approach to their design, which relies on model driven engineering, executable models and FMI based cosimulation. This approach is illustrated on the use case of an insular power grid and allows to study the impact of power production decisions.
\end{abstract}

\section{INTRODUCTION}

Smart Grids are the oncoming generation of power grids, enabled by information and communication technologies, taking part into the transformation of the electrical power landscape. They especially support the introduction of decentralized and renewable energy sources in the electrical production, allow prevention, better reactivity and improved response to events such as electrical failures. Smart Grids are therefore large-scale critical systems potentially impacting a lot of people. Such systems require thorough verification and validation before their implementation, and simulation is very valuable to evaluate various behavioral assumptions. Because they involve many interdependent technical domains, namely electronics, software information processing and telecommunications, Smart Grids are a typical example of complex systems to design. Model Driven Engineering (MDE) principles are well-suited to address the design and development issues of complex industrial systems by reasoning on executable models all along their life cycle (Hutchinson et al., 2011). However, there lacks a general approach to interconnect models from different technical domains in a cosimulation approach for engineering complex systems (Gomes et al., 2018). These models are designed using different tools for manipulating state machines, activity diagrams, discrete events or statistical models, and the accuracy and the predictive value of the cosimulation depend on a proper integration of these tools and on the synchronization of their execution. It is therefore mandatory to ensure the macroscopic alignment of the models with the business processes, and to maintain the consistency of the global model through the iterations of the individual models toward a final design.

This paper proposes an approach to assist Smart Grid designers in evaluating the global behavior of their solution and to evaluate the impact of energy production decisions. Our approach is based on MDE principles and on the FMI standard for the cosimulation of dynamic models, and focuses on computational and applicative modeling.

\section{SIMULATION OF CYBER-PHYSICAL SYSTEMS FOR THE INDUSTRY}

Our motivation is to cosimulate the different domains of a Smart Grid: electronics, software information processing and telecommunications, in order to evaluate its global behavior.

We identify specific requirements for the simulation of Smart Grids that can be considered as industrial cyber-physical systems. Different levels of abstraction in the models should be supported as the system may be too complex for extensive modeling. Because of that complexity, the knowledge of the global system is shared between several experts. Our proposition enforces separation of concerns and ensures 
the consistency of the interfaces between the various technical domains. Additionally, our work targets industrial contexts which require intellectual property protection and calls for solutions able to manipulate black-box models in a cosimulation approach.

Model Driven Engineering (MDE) is an approach covering the whole life-cycle of a software based system, such as Smart Grids, using executable modeling (Hutchinson et al., 2011). Models are processable in order to perform automated manipulations like verifications, simulations or transformations. While a lot of MDE frameworks raise concerns about UI and usability (Abrahão et al., 2017), our approach enables the reuse of existing industry-standard simulators for each domain of the system.

Functional Mockup Interface (FMI) is a standard for the cosimulation of dynamic systems allowing the interconnection of several different simulators in an integrated execution (Blochwitz et al., 2011). An artifact compliant with FMI is called a Functional Mockup Unit (FMU) and is a black-box encapsulation of a model and its simulation engine. The format of FMUs protects intellectual property, which is mandatory in the industrial context of Smart Grids. FMI is a standard allowing the separation of domains by subdividing the model of a Smart Grid into several models and their proper simulation tool, ensuring therefore modularity and reusability (Gomes et al., 2018).

In addition, information technology (IT) aspects are not considered in practice, and there is no IT simulator as such. Enterprise Architecture (EA) aims to model information, software and technology, often through the decomposition in several point-of-view layers with different levels of abstraction. But the models are usually not executable, and even less simulable. We draw from previous work (Seghiri et al., 2016) for modeling and executing EA processes and extend it by focusing on modeling the IT system for FMI cosimulation and addressing the issues for interfacing the heterogeneous models of a Smart Grid.

\section{RELATED WORKS}

In the electrical energy community, the challenge of simulating Smart Grids is not new. (Yang et al., 2013) sets up an environment to cosimulate Smart Grids with distributed control. Their approach uses software and hardware-in-the-loop simulations, where real controllers interact with a Matlab simulation of the plant through UDP and TCP communications. They particularly addressed the issue of the adaptation between event-driven and continuous components.
(Nutaro, 2011) explains how a power grid simulator should be designed in order "to accommodate the requirement for interoperability". The author developed his own power grid simulator based on numerical algorithms instead of only equations to combine discrete and continuous simulations in one engine. Sensors, controllers and electro-mechanical components are all modeled and simulated with that engine and a component library written in $\mathrm{C}++$. The simulator also implements an interface for time management and the injection of discrete data at runtime. To integrate a communication simulator, the power grid models are wrapped in components for the OMNET++ or NS2 simulators. This approach is efficient but provides a very specific solution for the cosimulation of Smart Grids and therefore lacks of extensibility. New behaviors and control equipments are defined by writing $\mathrm{C}++$ code, so there is little support for managing the refinement of the components during design. Also, using code as models prevents the use of MDE techniques for generating the wrappers necessary to interconnect the different simulators and to check the consistency of the whole simulation.

(Li et al., 2011) developed a framework called VPNet for Smart Grid simulation. The VPNet framework provides a cosimulation coordinator implemented in C\# to interface the OPNET communication simulator with VTB, a simulator for power grids with automated control. The two simulators are also both extended with interface modules to allow exchanges with the coordinator. This approach takes only into consideration the communication and the power aspects of a grid. The control part is integrated in the electrical simulation and there is no support for the IT aspect, which goes beyond "classical" control in Smart Grids. Moreover, this approach is tied to two specific simulators while our goal is to build a framework allowing (with limited development effort) the integration of any simulator. This is necessary because companies have developed specific simulators for handling technical aspects such as transients, harmonics and unbalanced networks. These legacy tools capture business knowledge that can be used during the design and analysis of a Smart Grid.

(Rohjans et al., 2014) notes that current Smart Grid simulation environments generally focus on one domain of the system, and expresses the need for a fully integrated environment handling multi-agent control, and interactions between these agents and the power system components. The paper presents a list of requirements for appropriate Smart Grid simulation tools, such as using time-stepped simulations, or allowing different paradigms in the models that are integrated. The authors conclude that there is a gen- 
eral lack of interoperability, and particularly of "a standardized and lightweight simulator API to enable syntactic interoperability (how data is exchanged), a standardized semantic description to achieve semantic interoperability (what is the meaning of exchanged data), and a well-defined model-independent scenario description language (what components are to be simulated and how they are interconnected)".

The existing solutions for the simulation of Smart Grids show what is technically possible and the kind of results we can expect. However, we would like to go further and to provide support for an evolving simulation of Smart Grids, from abstract models with coarse physical behaviors, up to very detailed models of the power grid, the telecom network, and the smart control algorithms.

\section{DESCRIPTION OF THE GENERAL APPROACH}

Our approach allows design engineers to simulate the behavior of their solutions, confronted to several scenarios. Based on the results, they can iterate at lower costs using different leverages in their models, such as equipment sizing, topology, or software algorithms.

\subsection{FMI Cosimulation}

The composition of a dynamic model and its simulator is called a simulation unit. A cosimulation is the interconnexion of several simulation units through their inputs and outputs, in order to obtain the trace of the evolution of every exposed variables in the different simulation units: the behavioral trace. The FMI standard defines a common interface to interact with a simulation unit, which is then called an FMU. It also constraints the data type of its inputs/outputs to 5 specific types: BOOLEAN, INTEGER, REAL, STRING or ENUMERATION. Our approach aims to execute a cosimulation integrating the FMUs produced by each domain expert involved in the Smart Grid design.

\subsection{Ensuring Proper Interconnection Between FMUs}

One of the main advantages of using a cosimulation environment is to allow the different experts to develop their own model in autonomy, with a minimal interference and in parallel with the others (Gomes et al., 2018). The choice of the FMI standard ensures the technological compatibility of each simu- lation unit, or FMU, with the cosimulation environment, without having to develop a specific connector to interface them.

However, the domain experts must share the same vision of the global design of the Smart Grid and its expected behavior, to ensure the relevance of their model and the accuracy of the simulation results. Hence all experts have to gather before the modeling phase in order to reach an agreement on a particular system design, and the distribution of concerns between the potential domain models.

We want to integrate and formalize this step of discussion in our approach, in order to ease the transition with the next steps of modeling and simulation. Therefore the first step of our approach is to make the different actors produce together a document called Inter-domain connections description. This document represents all possible interactions between the different domains, by expressing the static connections between the future FMUs, in compliance with FMI concepts such as data types. Hence each expert has complete authority on the modeling paradigm or technology, but must generate a FMU of its domain implementing the precise inputs and outputs defined in the document called Inter-domain connections description.

\subsection{A 4-Steps Iterative Cycle to Converge Toward an Acceptable Design}

We decompose the approach into a sequence of steps, parts of an iterative cycle.

Our approach involves several roles, usually fulfilled by different actors: the project leader has the global knowledge of the system, the stakes and objectives, and expresses the requirements, the domain experts develop the models of their own domain to simulate, and generate the corresponding FMU, the cosimulation architect configures and executes the cosimulation.

Each step is described by its objective, its method, its inputs and outputs.

1 - Inter-domain connections description: The step takes as input a global knowledge of the purpose and of the stakes of the system to design. It produces the Inter-domain connections description.

2 - Domain Modeling: The step takes as input the Inter-domain connections description. Each domain expert develops its model and produces a FMU for his domain, compliant with the data interfaces defined in this document. 
3 - Cosimulation: The step takes the Inter-domain connections description as input, and all the domain FMUs from the previous step. The Cosimulation Architect deploys the cosimulation according to the interconnections defined in the document, and executes it. It produces the behavioral trace of the system.

4 - Trace analysis \& iteration decisions: The step takes the behavioral trace from the simulation step as input. Various criteria are evaluated (like performance, efficiency, critical threshold) to determine if the solution is acceptable or not.

\section{APPLICATION TO A USE CASE AND MODELING OF THE IT BEHAVIOR}

\subsection{Use case: Islanded Smart Grid}

We chose a real use case from the French power utility to illustrate our contribution and apply our approach. It is an island which has an independent power grid from the mainland, with its own production equipments. A diesel power plant is the main energy producer, and is complemented by a photovoltaic farm. The main issue in the configuration lies in the reliability of the renewable energy supply. Indeed, as the photovoltaic source relies on sunlight and needs a clear sky for its production, it makes it as inconsistent and unpredictable as the weather. In order to balance the production with the consumption, it has to be sometimes forced to produce below its optimum, causing economic loss and carbon footprint degradation. Therefore, a chosen solution is to add a battery storage to damp the variability of the production, with the purpose of minimizing the limitations of the photovoltaic farm.

The efficiency of the system requires an Energy Management System (EMS) coupled with a Supervisory Control And Data Acquisition (SCADA) in order to implement an intelligent control of the production. The target infrastructure of the grid is shown in Figure 1.

\subsection{Interface Between Electric and IT Domains (Step 1)}

The project leader makes sure all the domain experts understand the objectives of the functionalities expected for the deployed system. Through discussion and from the chosen design, the actors will identify

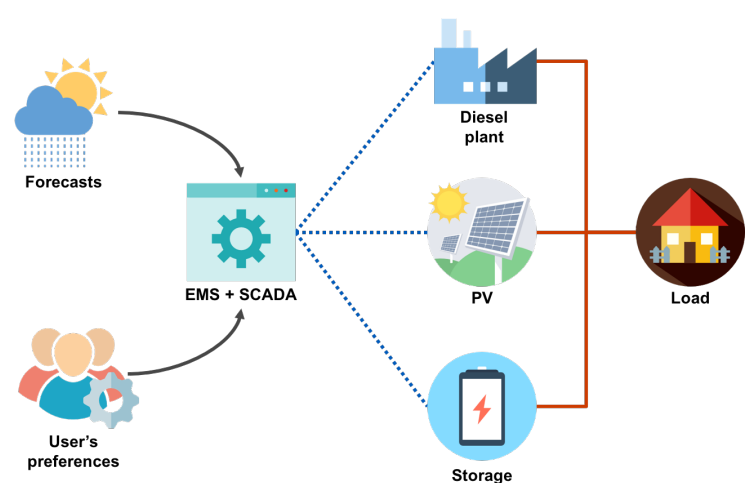

Figure 1: Target infrastructure of the "Islanded Smart Grid" use case

the simulation needs, the domains to model, and the nature of the interactions between the considered domains. We define an interaction as an exchange of data, or a behavioral instruction. These interactions are flattened into static connections complying with the FMI constraints by the cosimulation architect, and finally registered into the Inter-domain connections description.

For the "Islanded Smart Grid" use case presented above, we want to verify if a chosen design is operable and effective. In this paper, we consider only the IT and the electrical domains, ignoring the telecom network interaction. We come up with the following list of interactions between the two domains:

- The grid's equipments are sending their statevalues to the EMS (PV maximal production, stateof-charge of the battery, etc.), and the EMS sends in return control signals (PV-production's limitation, battery's power production or consumption, etc.).

- The EMS can trigger a switch between the coupled state (the diesel plant is connected to the grid and is the main source) and the islanded state (the diesel plant is shut down, and the supply is provided only by the photovoltaic farm and the battery). Presented in this way, the interaction has a discrete variability (the EMS punctually triggers the change of state), non-detectable during FMI cosimulation. It is easy to deduce a connection carrying a piecewise continuous variable: Grid_State_control either carries the coupled or islanded value.

Therefore, the Inter-domain connections description should look as shown in Figure 2. 


\begin{tabular}{|c|c|c|c|c|}
\hline Connection name & Unit & FMI Type & From & To \\
\hline PV_Max_Power & Watt & Real & Electric & IT \\
\hline PV_Limitation_control & Watt & Real & IT & Electric \\
\hline Battery_SOC & $\%$ & Real & Electric & IT \\
\hline Battery_Power & Watt & Real & Electric & IT \\
\hline Battery_Power_control & Watt & Real & IT & Electric \\
\hline Diesel_Power & Watt & Real & Electric & IT \\
\hline Grid_State_control & $\varnothing$ & $\begin{array}{c}\text { Enumeration } \\
\text { ["coupled", "islanded"] }\end{array}$ & IT & Electric \\
\hline
\end{tabular}

Figure 2: Use case: Inter-domain connections description

\subsection{Electrical Domain Model and FMU (Step 2)}

We developed a model of the electrical grid with the Modelica language (Chilard et al., 2015). Modelica is an object-oriented language used for the modeling of component-based systems. It is a declarative language where classes mainly contain sets of equations, defining the behavior of the system with no predefined causality (equality rather than assignment). Modelica is well suited for the modeling and simulation of physical systems like electrical circuits. Moreover the main simulation engines, OpenModelica ${ }^{1}$ and Dymola $^{2}$, implement the FMI standard, allowing the use and the generation of FMUs.

Our model computes all the electrical values in every point of the grid, and the state of all the electrical components (battery, plant, etc.). Its inputs implement the Inter-domain connections description defined in Figure 2, and it exposes the wanted variables as outputs. We finally exported our model into the FMU format to obtain the Electrical Domain FMU

\subsection{IT Domain Model and FMU (Step 2)}

As said in section 2, there is a lack of industrial simulation tool considering the IT aspects of a system. Some computational behavior can be evaluated through algorithms inserted inside models which support arithmetic operations, or executable scripts implemented with a programming language and linked with specific connectors or API to the rest of the simulation. But this nested configuration is difficult to maintain, so it is preferable and more aligned with MDE concepts to work with a consistent model of the IT behaviors of the system. The IT system is itself a complex sub-system of a Smart Grid. It represents all the ways the information is created, processed and used, through software or non-software components.

\footnotetext{
1 openmodelica.org

${ }^{2}$ www.3ds.com
}

\subsubsection{Modeling Method for the IT sub-system}

We propose here a method in two sub-steps to model the IT aspects of the Smart Grid, by developing several models with different levels of details. Hence several IT experts are involved in this development. The IT process expert is specialized in design and IT architecture, whereas the IT applicative expert is more specialized in computational behaviors, and software development.

Sub-step 1 : IT Process Modeling The IT system has several functions implemented by the various software applications of its composition. Each IT process describes how the IT system behaves in order to achieve a global objective, by making explicit the chain of functions involved in the realization of the objective. This sub-step consists for the IT process expert in developing the IT process model, a high level representation of the system, giving structure for its future refinements. The purpose is to allow one or several IT applicative experts to work on specific parts of the refined model while ensuring the consistency of the whole IT domain model.

The IT process model represents the relevant IT processes involved in the situations to simulate. It identifies the sequence of functions composing the different IT processes and their prototype (inputs and outputs), and makes the IT domain interface explicit. The chosen modeling language should have enough expressivity to model deterministic behaviors, events detection and generation, should allow composite complex behaviors from simple behaviors, and must be understandable by non-IT-process experts.

The IT process expert characterizes the functions of the system, arranges and coordinates them to build the models of the IT processes. An IT process in the IT process model can be triggered by internal or external events and must define the connections between the inputs and outputs of the functions. The IT domain interface is explicited and made accessible to the functions which need to interact with the outside of the IT domain.

Sub-step 2: IT Domain FMU The IT process model from the previous sub-step can not be simulated yet. The goal of this sub-step is to refine this model with the applicative behavior, in order to obtain a simulable model of the whole IT domain, and be able to generate an FMU: the IT domain FMU.

The IT applicative experts are in charge of modeling the application behaviors, to refine the IT process model to a Simulable IT process model. They develop one Function model for each function defined 
in the IT process model, the inputs and outputs being already defined. Then these models must be operated with the IT process model in one executable artifact, to generate the IT domain FMU.

For simple behaviors, the Function models may use the same language as the IT process model, hence be integrated inside this model thanks to the behavior decomposition ability of the language, constituting a Simulable IT process model. However, from our experience a language that is adapted to process modeling may not be relevant to describe complex application behaviors. Textual languages usually require more skills from the model developer but compensates with a lot more flexibility, efficiency and accuracy. FMI is precisely appropriate to execute heterogeneous and multi-paradigm codependent models through cosimulation. Therefore it may help in this case to constitute a consistent IT domain model, transposable to FMU and constituted from several FMU itself.

In this configuration, the IT process FMU generated from the Simulable IT process model would be the main orchestrator of the IT domain model execution. It would coordinate the execution of the Function models, whether they are part of the IT process $F M U$, or out-sided into their own FMU.

The several FMUs are connected together through a cosimulation scenario, and constitute a cosimulation unit. In order to be consistent with the expected output of the whole modeling step and produce a unique FMU for the domain, the IT Domain FMU, this cosimulation unit is converted to the FMU format. This operation may require the skills of the Cosimulation expert (cf. section 4).

\subsubsection{The "Islanded Smart Grid" Use Case}

IT Process Modeling In our example, we have one IT process, called Control the production, which implements the objective of maximizing the renewable part in the energy mix. We chose the $\mathrm{fUML}^{3}$ language for our model, which has enough expressivity to model deterministic behaviors, events detection and generation, and allow the composition of complex behaviors from several simple behaviors. fUML is an executable subset of the well-known UML standard, acknowledged for its graphical simplicity, hence understandable for non IT process experts.

We model the process by an fUML "activity diagram", an "activity" being seen as a process, and an "action" being seen as a function. The inputs and outputs of the functions are fUML objects.

The ITInterface object specifies the IT domain interface defined in the Inter-domain connections de-

\footnotetext{
${ }^{3}$ www.omg.org/spec/FUML
}

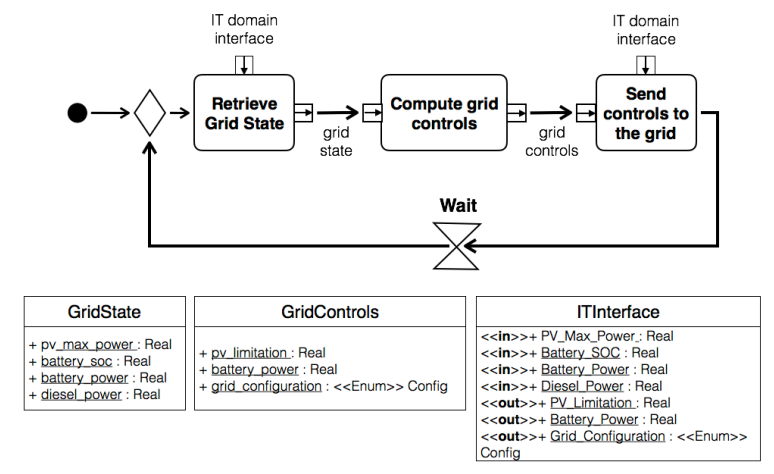

Figure 3: Use case: IT process model in fUML

scription, figure 2 . It is based on stereotypes to make the direction (in/out) of the data explicit. Figure 3 shows the two diagrams, class and activity, representing our IT process model for the "Islanded Smart Grid" use case.

IT Domain FMU The functions from the IT process model are modeled using "opaque actions", which are not executable in the fUML standard. The first objective of the IT applicative experts is therefore to develop the executable behavior of these functions in function models. Then the models are made compatible with FMI and the IT domain FMU is generated.

The Papyrus tool (Guermazi et al., 2015) and its plugin named Moka supports the fUML simulation and FMU export. In this regard, the ITInterface class is stereotyped with Moka's embed "FMU" profile, while its attributes are stereotyped with the "FMI port" profile. The FMU generator of Papyrus will use this class to build the interface of the FMU.

Both Retrieve Grid State and Send controls to the grid functions from the IT process model (Figure 3) are modeled directly into this model by two fUML activity diagrams (behavior composition). It mainly consists in accessing and manipulating the ITInterface's attributes, which will be the inputs / outputs of the to-be-generated IT Process FMU.

The Compute grid controls function model relies on an algorithm that manipulates data, and is more adapted to a textual language. We developed it in Java, and used the JavaFMI ${ }^{4}$ library to implement the FMI standard and generate the FMU. As a first implementation of an algorithm, we keep it simple and naive. It implements a decision table to determine if the battery is consuming or producing, if the PV production is limited or maximal, and we do not take any production or consumption forecasts into account.

In order to make the two models interact with each

\footnotetext{
${ }^{4}$ https://bitbucket.org/siani/javafmi
} 


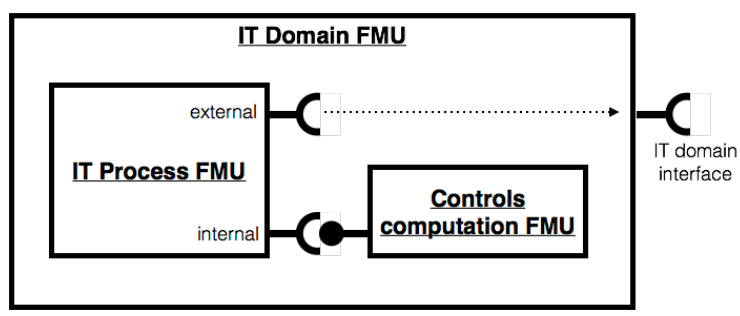

Figure 4: Use case: IT domain FMU composed of the IT process FMU and one out-sided function FMU

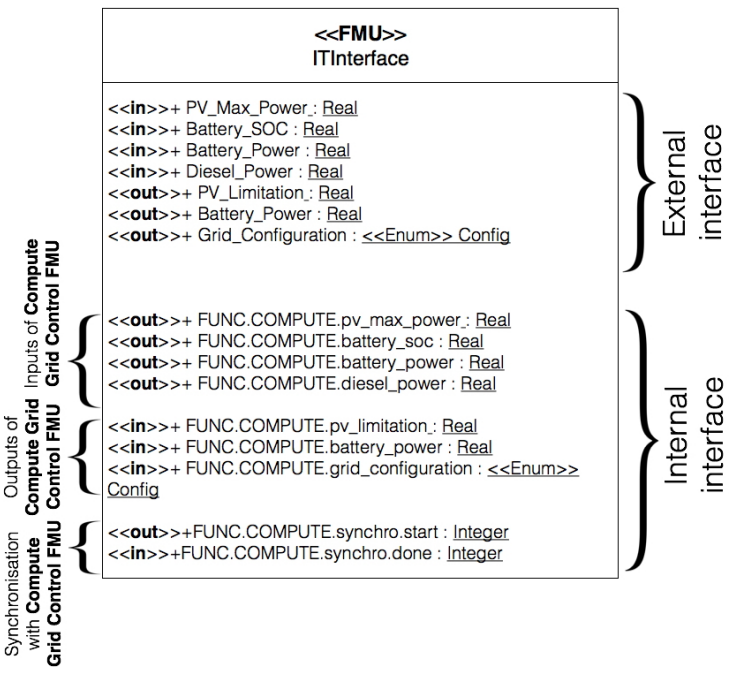

Figure 5: Use Case: ITInterface class of the Simulable IT process model

other during the simulation, we must implement in both models the corresponding FMI ports.

The DACCOSIM software (Tavella et al., 2016) is a simulation tool dedicated to FMI cosimulations. It is handling the composition of FMI cosimulation scenarios, and can produce an FMU from a cosimulation unit which is already composed of FMUs. Then the IT Process FMU and the Compute grid controls $F M U$ are connected together through a FMI cosimulation scenario in DACCOSIM, and encapsulated into a unique FMU exposing the interface of the IT domain (as shown in Figure 4), the IT Domain FMU.

The IT Process FMU exposes FMI ports corresponding to the IT domain, and FMI ports dedicated to the interaction with the out-sided function models, here the Compute grid controls function model.

Figure 5 shows the ITInterface class of the Simulable IT process model with these new ports.

\subsection{Cosimulation and Trace Analysis (Steps 3 \& 4)}

The IT Domain FMU and the Electrical Domain FMU are now generated and available for cosimula-

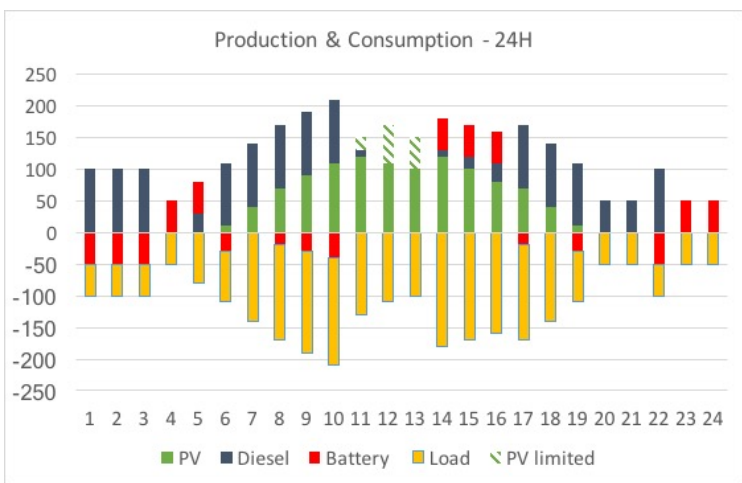

Figure 6: Use Case: Production and Consumption on a day

tion. The Cosimulation Architect defines the cosimultion scenario by configuring how these FMUs are connected, as described in the Inter-domain connections description. He or she also has to set the various parameters of the simulation: the starting and ending time, the step-size strategy (constant, adaptive) to balance between precision and computation length, the initial inputs and outputs values if not yet defined... Again we chose the DACCOSIM software to execute the cosimulation, as its master (algorithm) of cosimulation shows interesting functionalities improving the precision of the results (events detection, inteligent time step-sizing strategies, etc.).

The goal is to evaluate the design choices made by the design team. In our case, we are evaluating how the addition of a battery and an EMS improve the management of the production and the ratio of "green" energy used. Results should be assessed against quantified requirements in order to classify the solution either as "effective" or "non-effective".

Our scenario simulates the normal operation of the grid on a one day period, for a particular load curve. Figure 6 represents some of the simulated results, showing the variations of the consumption and of the production between the different power sources of the island (above the horizontal axis is the "production" and below is the "consumption").

The energy balance has been ensured all day (no black-out), but the PV production has been "limited" around midday (hatched bars). When we look at other results we see that the battery was saturated at this time. There is consequently a clear optimisation potential for our design, either the capacity of the battery is too low, or the EMS algorithms have to be improved. For our exemple, we know that the design of the EMS could be strongly improved by taking into account weather and consumption forecasts. We should iterate on the current design of the EMS model, and execute the cosimulation again to visualize directly the effect on the results. 


\section{CONCLUSION}

In this paper, we propose a cosimulation approach and highlight the purpose of its steps and how they are related. We provide insights for the realization of each step, by giving tool propositions and illustration with a use case. This use case is only a proof of concept, so the models and behaviors are kept simple. We should also perform further simulations with different parameters (load curves), over longer period to be really relevant on the assessment of the design choices.

In addition we are focusing on the IT Domain, by expliciting a method to model its behavior at different levels of detail to manage potential complexity.

We are planning to integrate the Telecom Domain in a next prototype. We are currently working on increasing the consistency of our approach, by enabling automated transitions between the different steps, in order to align more properly with the MDE guidelines. The first step on Inter-domain connections has a strong potential for improvement in this area. Executable models are the primary artifacts of MDE approaches, but the Inter-domain connections description produced in our approach is not executable. It only gives a hint to the modelers on how to make the interactions between the models, but nothing prevents them to do otherwise, or to be mistaken. Moreover, the fact that all the actors reach an agreement on the final interface between domains very early in the design process when no model has been defined yet is a strong hypothesis of our approach.

Future evolutions of our approach rely on a highlevel architectural model, like the one exposed in (Andrén et al., 2017), from which domain interfaces and interconnections can be deduced automatically. Consistency verification with domain models and automated transformations should also be facilitated. Finally, the simulation of a Smart Grid is relevant if we can ensure that the future implementation and deployment of the system conform to its models.

\section{REFERENCES}

Abrahão, S., Bourdeleau, F., Cheng, B. H. C., Kokaly, S., Paige, R. F., Störrle, H., and Whittle, J. (2017). User Experience for Model-Driven Engineering: Challenges and Future Directions. In MODELS 2017.

Andrén, F., Strasser, T., and Kastner, W. (2017). Engineering Smart Grids: Applying Model-Driven Development from Use Case Design to Deployment. Energies, 10(3):374.
Blochwitz, T., Otter, M., Arnold, M., Bausch, C., Elmqvist, H., Junghanns, A., Mauss, J., Monteiro, M., Neidhold, T., Neumerkel, D., and others (2011). The Functional Mockup Interface for Tool Independent Exchange of Simulation Models. In 8th Int. Modelica Conf., Dresden, Germany, pages 105-114.

Chilard, O., Boes, J., Perles, A., Camilleri, G., Gleizes, M.-P., Tavella, J.-P., and Croteau, D. (2015). The Modelica Language and the FMI Standard for Modeling and Simulation of Smart Grids. pages 189-196.

Gomes, C., Thule, C., Broman, D., Larsen, P. G., and Vangheluwe, H. (2018). Co-simulation: A survey. ACM Computing Surveys, 51(3):49:149:33.

Guermazi, S., Tatibouet, J., Cuccuru, A., Dhouib, S., Gérard, S., and Seidewitz, E. (2015). Executable modeling with fUML and alf in papyrus: Tooling and experiments. In EXE@MoDELS.

Hutchinson, J., Rouncefield, M., and Whittle, J. (2011). Model-driven engineering practices in industry. In ICSE 2011, pages 633-642.

Li, W., Monti, A., Luo, M., and Dougal, R. A. (2011). VPNET: A co-simulation framework for analyzing communication channel effects on power systems. In 2011 IEEE Electric Ship Technologies Symposium, pages 143-149.

Nutaro, J. (2011). Designing power system simulators for the smart grid: Combining controls, communications, and electro-mechanical dynamics. In 2011 IEEE Power and Energy Society General Meeting, pages 1-5.

Rohjans, S., Lehnhoff, S., Schütte, S., Andrén, F., and Strasser, T. (2014). Requirements for Smart Grid simulation tools. In ISIE 2014, pages 17301736.

Seghiri, R., Boulanger, F., Lecocq, C., and Godefroy, V. (2016). An Executable Model Driven Framework for Enterprise Architecture Application to the Smart Grids Context. In HICSS 2016, pages 4546-4555.

Tavella, J.-P., Caujolle, M., Vialle, S., Dad, C., Tan, C., Plessis, G., Schumann, M., Cuccuru, A., and Revol, S. (2016). Toward an Accurate and Fast Hybrid Multi-Simulation with the FMI-CS Standard. In ETFA 2016, Berlin, Germany.

Yang, C., Zhabelova, G., Yang, C., and Vyatkin, V. (2013). Cosimulation Environment for EventDriven Distributed Controls of Smart Grid. IEEE Trans. Industrial Informatics, 9(3):1423-1435. 